\title{
Chronic hypersensitivity pneumonitis; an enigmatic and frequently fatal disease
}

\author{
Toby M. Maher ${ }^{1,2,3}$
}

Affiliations: ${ }^{1}$ Hastings Centre for Pulmonary Research and Division of Pulmonary, Critical Care and Sleep Medicine, Keck School of Medicine, University of Southern California, Los Angeles, CA, USA. ${ }^{2}$ NIHR Respiratory Clinical Research Facility, Royal Brompton Hospital, London, UK. ${ }^{3}$ National Heart and Lung Institute, Imperial College, London, UK.

Correspondence: Toby Maher, Hastings Centre for Pulmonary Research and Division of Pulmonary, Critical Care and Sleep Medicine, Keck School of Medicine, University of Southern California, 2020 Zonal Avenue, Los Angeles, CA 90033, USA. E-mail: t.maherdimperial.ac.uk

@ERSpublications

Chronic hypersensitivity pneumonitis is a poorly understood condition. The many unknowns encompass pathogenesis, diagnosis, disease behaviour and treatment. Without international collaborative research it will remain an enigmatic but fatal disease. https://bit.ly/2BISI6k

Cite this article as: Maher TM. Chronic hypersensitivity pneumonitis; an enigmatic and frequently fatal disease. Eur Respir Rev 2020; 29: 200177 [https://doi.org/10.1183/16000617.0177-2020].

In the current issue of the European Respiratory Review, CREAMER and BARRATT [1] review prognostic factors in chronic hypersensitivity pneumonitis (cHP). Their work provides a state-of-the-art overview on the topic; but in doing so, highlights many of the challenges confronting patients and clinicians dealing with this enigmatic condition. Many important knowledge gaps exist pertaining to cHP which impact everything from understanding of the mechanisms underpinning pathogenesis, disease natural history, optimal approaches to diagnosis, how to distinguish cHP from idiopathic pulmonary fibrosis (IPF) and best treatment.

Hypersensitivity pneumonitis was first described in the early part of the 18th century by Italian physician Bernadino Ramazzini who recognised the symptoms of the disease in a cohort of grain workers [2]. In the intervening three centuries the basic pathophysiology of the disease has been disentangled and a multitude of domestic, environmental, iatrogenic and industrial triggers have been identified. Ultimately, hypersensitivity pneumonitis arises due to an aberrant immunological response to respirable, usually organic, particles which trigger a granulomatous lymphocytic alveolitis and bronchiolitis. Historically, the condition has been categorised into acute, sub-acute and chronic variants. Although the basic pathobiology underpinning development of both acute and chronic forms of hypersensitivity pneumonitis is assumed to be the same, it is unknown exactly how the two forms of the disease relate to one another. Are individuals who develop acute hypersensitivity pneumonitis at risk of developing cHP? Are age or burden or duration of antigen exposure important determinants for the development of cHP? Genetic variants which increase the risk for developing IPF, including single nucleotide polymorphisms (SNPs) in genes related to the telomere complex, also predispose to the development of cHP [3]; suggesting that the immunological and fibrotic components of the disease are probably independent and separately regulated.

At face value, the diagnosis of cHP should be straightforward. Clinically, it is anticipated that patients with cHP will have a history of exposure to a relevant respirable antigen. In reality, diagnosis is frequently challenging. Over half of individuals diagnosed with cHP lack an overt history of a relevant exposure [1]; whether this reflects misdiagnosis, cryptic exposure to antigen or the existence of antigens hitherto not

Provenance: Commissioned article, peer reviewed

Received: 2 June 2020 | Accepted after revision: 27 June 2020

Copyright CERS 2020. This article is open access and distributed under the terms of the Creative Commons Attribution Non-Commercial Licence 4.0. 
recognised to cause hypersensitivity pneumonitis remains unknown. Histologically, hypersensitivity pneumonitis is characterised by the presence of airway-centred granulomata, peri-bronchial metaplasia, lymphocytic bronchiolitis and varying degrees of interstitial fibrosis. These histological changes are reflected on computed tomography imaging by the presence of airway-centred fibrosis, gas-trapping and ground-glass attenuation. The imaging appearances of cHP lack specificity and overlap with the appearances seen in conditions such as IPF, fibrotic sarcoidosis and connective tissue disease-associated interstitial lung disease (ILD). Similarly, at a histological level, the changes seen in cHP overlap with other fibrotic and granulomatous diseases. Furthermore, airway-centred granulomata can represent a localised response to injury by inhaled particles. Thus, the relevance of very low numbers of granulomata in fibrotic disease otherwise resembling IPF remains unknown. These uncertainties impact the confidence with which clinicians are able to diagnose cHP and are exacerbated by an absence of any internationally accepted guidelines on diagnosis. A recent multi-national study assessing the role of the multi-disciplinary team in diagnosing ILDs found very poor diagnostic agreement for cases of possible cHP; the level of agreement was far worse than that seen for IPF or connective tissue disease-associated ILD [4].

When it comes to accurately diagnosing cHP the most challenging differential diagnosis is that of IPF. Although there are prognostic differences between IPF and cHP, until a decade ago, achieving an accurate diagnosis was, in most cases, an academic exercise as treatment of both disorders was similar. This changed following the observation that high-dose corticosteroids and azathioprine are deleterious in IPF and the approval of pirfenidone and nintedanib [5]. Consequently, the distinction between cHP and IPF has become an important one. There are concerns both that cHP is misdiagnosed as IPF, thus denying patients access to potentially effective therapies, and vice versa that IPF is misdiagnosed as cHP leading to patients being treated with harmful immunosuppressive regimens. An alternative possibility is that IPF represents the end-consequence of repeated damage to the aged lung and that any disorder causing repeated epithelial injury (be it immunological as in hypersensitivity pneumonitis or through direct injury as occurs in asbestosis) can lead to the same final common outcome of progressive, irreversible fibrosis [6]. If this is the case, cHP might respond differently to specific therapies based on stage of disease and the balance between active inflammation driven by ongoing immunological response to inhaled antigen and fibrogenesis. Without a detailed understanding of the true natural history of cHP such theories remain highly speculative but are important in determining best management of both diseases.

Leaving aside the intersect between $\mathrm{CHP}$ and IPF, another critical unknown is the optimal therapy for cHP. Antigen avoidance is presumed to be important [1]. However, evidence that avoidance modulates disease progression in cHP is scanty. Furthermore, as noted, many individuals with cHP either have no identifiable exposure history or else have exposures of unknown relevance, e.g. to feathers in bedding or cushions. Thus, offering lifestyle advice, especially advice that impacts an individual's work or that necessitates major changes to the domestic environment, is challenging given that it is not certain that even removal of an identifiable exposure alters prognosis. Another controversial area in the treatment of cHP is the role of corticosteroids and immunosuppressants. In acute hypersensitivity pneumonitis, specifically farmers lung, corticosteroids have been shown, in a small placebo-controlled trial, to speed up symptomatic and radiographic resolution of hypersensitivity pneumonitis [7]. However, by 12 months there was no difference between those individuals who received corticosteroids or placebo. This observation together with the knowledge that aberrant immune activation underpins the pathogenesis of cHP has led to corticosteroids and immunosuppressants such as azathioprine, mycophenolate mofetil and cyclophosphamide being widely used as treatment for cHP. There is some observational and retrospective evidence that such strategies may be beneficial in individuals with a lymphocytic infiltrate on bronchoalveolar lavage [8]. However, none of these therapies have been tested in a randomised control trial in cHP. Until such time as they are, there remains an important concern that, as is the case in IPF, aggressive immunosuppression in individuals with established pulmonary fibrosis due to cHP may be harmful. More recently, nintedanib has been shown to be effective at slowing disease worsening in individuals with progressive fibrosis due to any cause [9]. Approximately a quarter of subjects in the trial had a diagnosis of cHP. Similarly, a study of pirfenidone in unclassifiable ILD (where the majority of diagnostic uncertainty arose because of the difficulty of discriminating cHP from IPF) demonstrated an important effect of treatment on slowing disease progression [10]. Taken together, these data suggest that anti-fibrotic therapy is effective in slowing decline in at least a subset of individuals with cHP. Without further studies, however, it is impossible to know which treatments should be considered first line in cHP, whether immunosuppressants should be combined with anti-fibrotic drugs or what should be the sequencing or timing of the introduction of specific therapies.

CREAMER and BARRATT [1] conclude their review by calling for multicentre and international collaborative efforts to address the many unknowns in cHP. This point can only be reiterated. To change outcomes for individuals with $\mathrm{cHP}$ it is important that we better understand all facets of the disease from initial 
response to antigen through to terminal fibrosis. Without time, energy and resources being dedicated to cHP the disease will remain a fatal enigma.

Conflict of interest: T.M. Maher has received grants and personal fees from GlaxoSmithKline R\&D and AstraZeneca; personal fees from Boehringer Ingelheim, Roche, Bayer, Samumed, Galapagos, Celgene, Indalo, Pliant, Blade Therapeutics, Respivant, Novartis and Bristol-Myers Squibb, outside the submitted work. He has also received, via his institution, industry-academic funding from and has received consultancy or speakers' fees from Apellis.

Support statement: T.M. Maher is supported by a NIHR-funded Clinician Scientist Fellowship (NIHR Ref: CS-2013-13-017) and British Lung Foundation Chair in Respiratory Research (C17-3).

\section{References}

1 Creamer AW, Barratt SL. Prognostic factors in chronic hypersensitivity pneumonitis. Eur Respir Rev 2020; 29: 190167.

2 Ross D. Bernardino Ramazzini (1633-1714). Occup Health (Lond) 1979; 31: 136-141.

3 Ley B, Torgerson DG, Oldham JM, et al. Rare protein-altering telomere-related gene variants in patients with chronic hypersensitivity pneumonitis. Am J Respir Crit Care Med 2019; 200: 1154-1163.

4 Walsh SLF, Wells AU, Desai SR, et al. Multicentre evaluation of multidisciplinary team meeting agreement on diagnosis in diffuse parenchymal lung disease: a case-cohort study. Lancet Respir Med 2016; 4: 557-565.

5 Idiopathic Pulmonary Fibrosis Clinical Research N, Raghu G, Anstrom KJ, et al. Prednisone, azathioprine, and N-acetylcysteine for pulmonary fibrosis. N Engl J Med 2012; 366: 1968-1977.

6 Wolters PJ, Blackwell TS, Eickelberg O, et al. Time for a change: is idiopathic pulmonary fibrosis still idiopathic and only fibrotic? Lancet Respir Med 2018; 6: 154-160.

7 Kokkarinen JI, Tukiainen HO, Terho EO. Effect of corticosteroid treatment on the recovery of pulmonary function in farmer's lung. Am Rev Respir Dis 1992; 145: 3-5.

8 De Sadeleer LJ, Hermans F, De Dycker E, et al. Impact of BAL lymphocytosis and presence of honeycombing on corticosteroid treatment effect in fibrotic hypersensitivity pneumonitis: a retrospective cohort study. Eur Respir $J$ 2020; 55: 1901983.

9 Flaherty KR, Wells AU, Cottin V, et al. Nintedanib in progressive fibrosing interstitial lung diseases. N Engl J Med 2019; 381: 1718-1727.

10 Maher TM, Corte TJ, Fischer A, et al. Pirfenidone in patients with unclassifiable progressive fibrosing interstitial lung disease: a double-blind, randomised, placebo-controlled, phase 2 trial. Lancet Respir Med 2020; 8: 147-157. 